\title{
UNCOVERING THE OUTFLOW DRIVEN BY THE BROWN DWARF LS-RCrA 1: H $\alpha$ AS A TRACER OF OUTFLOW ACTIVITY IN BROWN DWARFS*
}

\author{
E. T. Whelan ${ }^{1}$, T. P. RAY ${ }^{1}$, AND F. BacciotTi ${ }^{2}$ \\ ${ }^{1}$ School of Cosmic Physics, Dublin Institute for Advanced Studies, Dublin, Republic of Ireland \\ ${ }^{2}$ Osservatorio Astrofisico di Arcetri, 50125 Firenze, Italy \\ Received 2008 November 16; accepted 2008 December 17; published 2009 January 12
}

\begin{abstract}
It is now apparent that classical $\mathrm{T}$ Tauri-like outflows commonly accompany the formation of young brown dwarfs (BDs). To date two optical outflows have been discovered, and results presented in this Letter increase this number to three. Using spectro-astrometry, the origin of the LS-RCrA 1 forbidden emission lines in a blueshifted outflow is confirmed. The nondetection of the redshifted component of the outflow in forbidden lines, along with evidence for some separation between low- and high-velocity outflow components, does not support the hypothesis that LS-RCrA 1 has an edge-on accretion disk. The key result of this analysis is the discovery of an outflow component to the $\mathrm{H} \alpha$ line. The $\mathrm{H} \alpha$ line profile has blueshifted and redshifted features in the wings which spectro-astrometry reveals to also originate in the outflow. The discovery that $\mathrm{H} \alpha$ emission in BDs can have a significant contribution from an outflow suggests that the use of $\mathrm{H} \alpha$ line widths as a proxy of mass accretion in BDs is not clear cut. This method assumes that any contribution to the $\mathrm{H} \alpha$ line flux from a possible outflow is negligible. Finally, the fact that the $\mathrm{H} \alpha$ line traces both lobes of the outflow while only the blueshifted lobe is seen in forbidden emission points to the presence of a dust hole in the accretion disk of LS-RCrA 1. This is commonly seen in classical $\mathrm{T}$ Tauri stars and is assumed to signal the onset of planet formation.
\end{abstract}

Key words: ISM: jets and outflows - stars: low-mass, brown dwarfs - stars: mass loss

\section{INTRODUCTION}

The study of brown dwarfs (BDs) in star-forming regions has a special importance as it not only allows the formation and evolution of $\mathrm{BDs}$ to be investigated but also addresses whether the low-mass paradigm for star formation can be extended into the substellar regime. Much of what is known about low-mass star formation, and in particular the connection between magnetospheric accretion and outflow activity, comes from studying the classical T Tauri stars (CTTSs; Edwards 2008; Ray et al. 2007). Observational studies point to strong similarities between the evolution of young BDs and CTTSs. To summarize, BDs are now known to have disks (Wilking et al. 1999; Natta \& Testi 2001; Natta et al. 2002), to exhibit T Tauri-like accretion activity and variability (Natta et al. 2004; Scholz \& Jayawardhana 2006), and to drive outflows. To date, two BD optical outflows (Whelan et al. 2005, 2007) and two molecular outflows (Phan-Bao et al. 2008; Bourke et al. 2005) have been reported. Further investigation is needed in order to properly compare $\mathrm{BD}$ outflows to protostellar outflows.

LS-RCrA 1 has a spectral type of M6.5 and an estimated mass of 35-72 $M_{\mathrm{J}}$ placing it within the BD mass range (Barrado y Navascués et al. 2004; Scholz \& Jayawardhana 2006). The $10 \%$ width of the $\mathrm{H} \alpha$ line is commonly used as a test of accretion activity in BDs. BDs with $10 \%$ width greater than $200 \mathrm{~km}$ $\mathrm{s}^{-1}$ (and equivalent width $>10 \AA$ ) are classified as accretors and below this as nonaccretors (Jayawardhana et al. 2003). The $\mathrm{H} \alpha 10 \%$ width and equivalent width (EW) clearly demonstrate that LS-RCrA 1 is an intense accretor (Scholz \& Jayawardhana 2006; Fernández \& Comerón 2001). Analysis of the spectra discussed here places the $\mathrm{H} \alpha 10 \%$ width between $270 \mathrm{~km} \mathrm{~s}^{-1}$ and

\footnotetext{
* Based on data collected by UVES observations (observing runs 67.C-0549(B), 69.B-0126(A), 71.C-0429(C), and 71.C-0429(D)) at the VLT on Cerro Paranal (Chile) which is operated by the European Southern Observatory (ESO).
}

$300 \mathrm{~km} \mathrm{~s}^{-1}$. Scholz \& Jayawardhana (2006) give the EW as varying between $44 \AA$ and $125 \AA$. Forbidden emission lines (FELs) have proved to be effective tracers of outflow activity in CTTSs and to date they have been used to explore the kinematics, morphology, and physical conditions of jets at high angular resolution (Ray et al. 2007). The so-called traditional tracers of CTT jets, i.e. [O I] $\lambda \lambda 6300,6363$, [S II] $\lambda \lambda 6716,6731$, and [N II] $\lambda 6583$, are found in the spectra of young BDs. This finding was the first indication that BDs commonly launch outflows. In addition to being an intense accretor, LS-RCrA 1 was the first BD shown to have FELs and notably it has the strongest forbidden emission of any BD studied to date (Fernández \& Comerón 2001, 2005).

A further interesting feature of LS-RCrA 1 is its subluminous nature. Fernández \& Comerón (2001) first identified this object and noted that its luminosity is far less than other objects of similar spectral type. LS-RCrA 1 is a strong accretor and current evolutionary models do not take accretion activity into account. Fernández \& Comerón (2001) argue that this could lead to an overestimation of the age of LS-RCrA 1, thus explaining the lack of agreement between its spectral type and luminosity. (Also refer to Tout et al. 1999.) An alternative explanation is that LS-RCrA 1 could have a near-edge-on disk, leading to its photosphere being predominantly seen in scattered light (Barrado y Navascués et al. 2004). As a consequence, it would appear fainter (and older) than it really is.

In this Letter, the results of a spectro-astrometric analysis of key lines in the spectrum of LS-RCrA 1 are discussed. Spectroastrometry has been used previously to find outflows driven by the BDs ISO-Oph 102 (Whelan et al. 2005) and 2MASS12073932 (Whelan et al. 2007). The molecular component to the ISOOph 102 outflow has recently been detected (Phan-Bao et al. 2008). Although LS-RCrA 1 has strong forbidden lines, no spatial extension in the line regions has been directly observed (Fernández \& Comerón 2005). Here, the displacement of the 

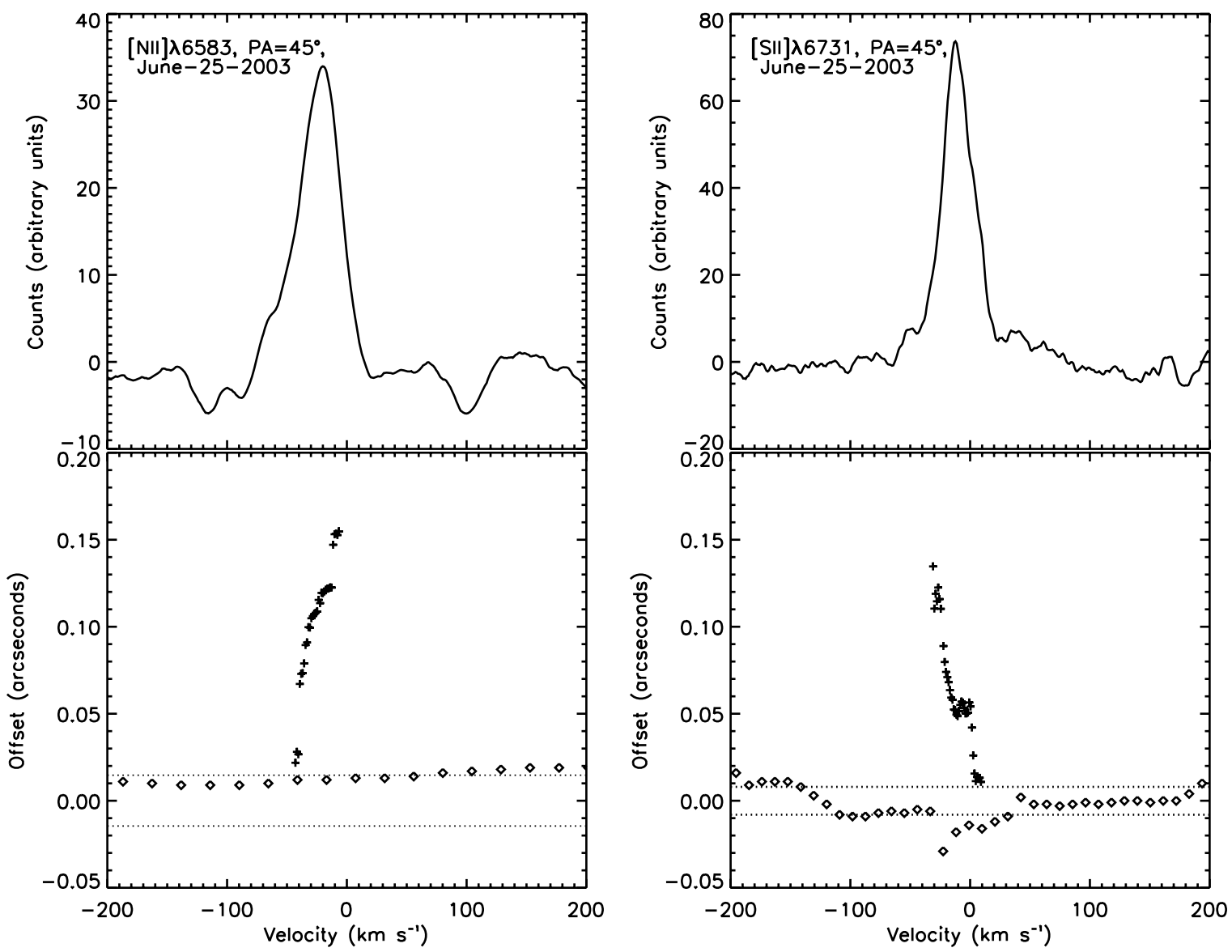

Figure 1. Spectro-astrometry of the blueshifted $\left[\mathrm{N}_{\mathrm{II}}\right] \lambda 6583$ and $\left[\mathrm{S}_{\mathrm{II}}\right] \lambda 6731$ lines at a slit P.A. of $45^{\circ}$. As explained, the spectra are summed in order to increase the $\mathrm{S} / \mathrm{N}$ and thus the spectro-astrometric accuracy. The diamonds represent the continuum position measured over a range of velocities, and the crosses the offset in the pure line region. All velocities are systemic. The accuracy in the measurement of both the line and continuum position is comparable and the dashed lines delineate the $\pm 1 \sigma$ error.

Table 1

Spatial Offsets Measured in the Blueshifted [N II] 6583 and [S II] 76731 Lines and the Blue/Red $\mathrm{H} \alpha$ Line Wings at Slit P.A.s between $45^{\circ}$ and $-73^{\circ}$

\begin{tabular}{|c|c|c|c|}
\hline P.A. (avg) & Date & Line & Maximum Offset (mas) \\
\hline $45^{\circ}$ & 2003 Jun 25 & $\begin{array}{c}{[\mathrm{N} \text { II }] \lambda 6548,[\mathrm{~S} \text { II }] \lambda 6731} \\
\mathrm{H} \alpha\end{array}$ & $\begin{array}{c}150 \pm 14,140 \pm 9 \\
60,-45( \pm 14)\end{array}$ \\
\hline $63^{\circ}$ & 2003 Jun 3 & $\begin{array}{c}{[\mathrm{N} \text { II }] \lambda 6548,[\mathrm{~S} \mathrm{II}] \lambda 6731} \\
\mathrm{H} \alpha\end{array}$ & $\begin{array}{c}100 \pm 25,110 \pm 12 \\
50,-28( \pm 7)\end{array}$ \\
\hline $74^{\circ}$ & 2003 Jun 9 & $\begin{array}{c}{[\mathrm{N} \text { II }] \lambda 6548,[\mathrm{~S} \text { II }] \lambda 6731} \\
\mathrm{H} \alpha\end{array}$ & $\begin{array}{c}100 \pm 24,100 \pm 12 \\
15,-15( \pm 5)\end{array}$ \\
\hline $82^{\circ}$ & 2003 Jun 13 & $\begin{array}{c}{[\mathrm{N} \text { II }] \lambda 6548,[\mathrm{~S} \text { II }] \lambda 6731} \\
\mathrm{H} \alpha\end{array}$ & $\begin{array}{c}40 \pm 5,45 \pm 9 \\
10,-10( \pm 4)\end{array}$ \\
\hline$-73^{\circ}$ & $2003 \mathrm{Jul} 4$ & $\begin{array}{c}{\left[\mathrm{N}_{\mathrm{II}}\right] \lambda 6548,[\mathrm{~S} \text { II }] \lambda 6731} \\
\mathrm{H} \alpha\end{array}$ & $\begin{array}{c}-80 \pm 19,-80 \pm 10 \\
-5,5( \pm 6)\end{array}$ \\
\hline
\end{tabular}

forbidden emission and $\mathrm{H} \alpha$ line wings from the BD position is mapped, confirming their origin in an outflow driven by LSRCrA 1. Results contribute to the debate over the cause of the subluminous nature of LS-RCrA 1, and increase the number of confirmed BD optical outflows to three.

\section{OBSERVATIONS AND ANALYSIS}

The high-resolution $(R=57,000)$ spectra presented here were obtained with the UV-Visual Echelle Spectrometer (UVES; Dekker et al. 2000) on the ESO VLT UT2 and first published by Fernández \& Comerón (2005). For the purpose of spectro-astrometric analysis, the raw data were obtained from the ESO Archive. Observations of LS-RCrA 1 were taken over a range of dates between 2003 June 3 and 2003 July 4 and at a range of slit position angles (P.A.s). As reported by Fernández \& Comerón (2005), the range of P.A.s resulted from the setting of the parallactic angle so as to minimize losses due to atmospheric differential refraction (refer to Table 1). The seeing over all nights varied between 0.9 and $1^{\prime \prime} .7$ and the spatial sampling was 0 '.182 per pixel. The data were analyzed using spectroastrometry as outlined in Whelan et al. (2005) and Whelan \& Garcia (2008).

Spectro-astrometry was first applied to the optical/nearinfrared spectra of Herbig Ae/Be and CTTSs (Bailey 1998; Whelan et al. 2004). Application of the technique to the study of low- and intermediate-mass stars is relatively straightforward as both the continuum and line emission are strong. However, in the case of BDs, the continuum emission in the range 6000-7000 is inherently faint and the FELs are far weaker than those excited in CTT jets. In order to increase the signal-to-noise ratio $(\mathrm{S} / \mathrm{N})$ of the $\mathrm{BD}$ spectra and thus the spectro-astrometric accuracy, the spectra are summed or smoothed (in the dispersion direction) before the transverse spatial profiles are fitted (Whelan et al. 2007). Also as explained in Whelan et al. (2005) and Whelan \& Garcia (2008), the continuum is fitted and then removed before the offset in the line region is measured. In our study of BDs, we sum/smooth the noncontinuum-subtracted and subtracted spectra separately so that the accuracy to which we measure the position of the line and continuum emission is comparable. The method chosen to increase the $\mathrm{S} / \mathrm{N}$ does not affect the overall results. In Figures 1 and 2, each point is the centroid 


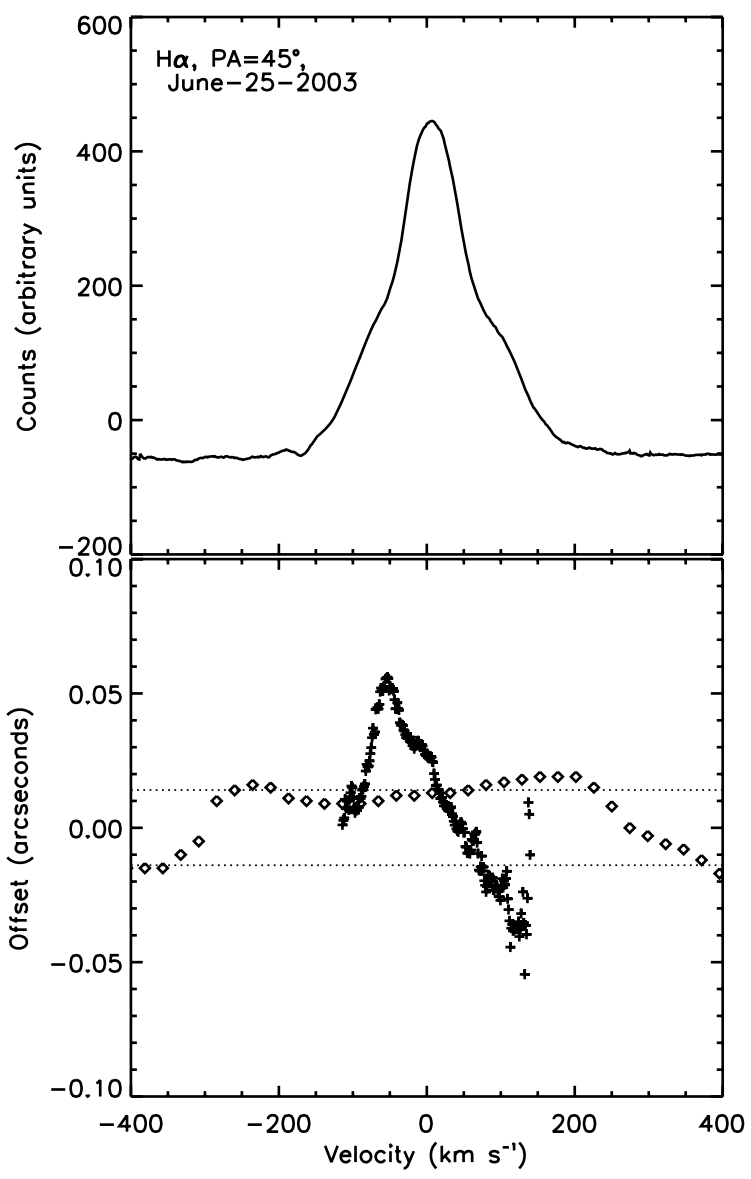

Figure 2. Spectro-astrometry of the $\mathrm{H} \alpha$ line at a slit P.A. of $45^{\circ}$. The spectroastrometric analysis was carried out in the same way as for the FELs. The LS-RCrA 1 outflow is clearly contributing significantly to the overall line flux, and offsets in the wings are revealed at velocities of $\sim-50 \mathrm{~km} \mathrm{~s}^{-1}$ and $+100 \mathrm{~km} \mathrm{~s}^{-1}$

of a spatial profile extracted at a particular velocity and each extracted profile is the sum of a number of adjacent profiles. Hence offsets are a moving sum across the continuum and continuum-subtracted line region.

In the case of LS-RCrA 1 , while a high $\mathrm{S} / \mathrm{N}$ was achieved for the forbidden emission and $\mathrm{H} \alpha$ lines, the continuum emission was relatively very faint (recall that LS-RCrA 1 is described as subluminous). Indeed, as reported in Whelan et al. (2006), spectra of LS-RCrA 1 obtained using the Magellan Inamori Kyocera Echelle (MIKE) on the Magellan II Telescope were found to be unsuitable for spectro-astrometric analysis, simply due to the nondetection of the continuum emission. Even with the better S/N UVES spectra obtained by Fernández \& Comerón (2005) and increasing the S/N (as described above), it was still only possible to recover the continuum position in the region of the $\mathrm{H} \alpha$, $\left[\mathrm{S}_{\mathrm{II}}\right] \lambda \lambda 6716,6731$, and [N II] 26583 lines but not at bluer wavelengths.

Also note that as in previous investigations of BD outflows with UVES, spectro-astrometric artifacts (Brannigan et al. 2006) were not found to be a problem. In Whelan et al. (2005, 2007), artifacts were ruled out through the demonstration that lines like He I $\lambda 6678$ were not offset. If measured offsets were indeed artifacts then displacements would be found in all lines, even those which are chromospheric in origin. In this study, spectra are analyzed at several P.A.s and overall measured offsets in the lines are on a scale of 50-200 mas indicating the presence of spatially extended emission originating in an outflow. Offsets vary with P.A. as expected, providing confirmation that they are real.

\section{RESULTS AND DISCUSSION}

Table 1 lists the maximum spectro-astrometric offsets in the blueshifted [N $\left.{ }_{\text {II }}\right] \lambda 6583$ and $\left[\mathrm{S}_{\mathrm{II}}\right] \lambda 6731$ lines and the blue/red $\mathrm{H} \alpha$ wings, for the range of slit P.A.s used. In addition, the spectro-astrometric analysis at $45^{\circ}$ is presented in Figures 1 and 2 . Offsets are with respect to the continuum position with positive offsets to the north and negative to the south. Fernández \& Comerón (2005) give the average velocities of the LS-RCrA 1 FELs at $\sim-5 \mathrm{~km} \mathrm{~s}^{-1}, \sim-22 \mathrm{~km} \mathrm{~s}^{-1}$, and $\sim-13 \mathrm{~km} \mathrm{~s}^{-1}$ for the $\left[\mathrm{O}_{\mathrm{I}}\right] \lambda 6300,\left[\mathrm{~N}_{\mathrm{II}}\right] \lambda 6583$, and $\left[\mathrm{S}_{\mathrm{II}}\right] \lambda 6731$ lines, respectively. Our reduction of their data yields the same average values. Uniquely, the outflow is also detected in $\mathrm{H} \alpha$. Blueshifted and redshifted "humps" are observed at velocities of $\sim-50 \mathrm{~km} \mathrm{~s}^{-1}$ and $+100 \mathrm{~km} \mathrm{~s}^{-1}$ and these are found to be offset in the direction of the blueshifted outflow and redshifted counterflow, respectively, as defined by the FELs (refer to Table 1 and Figure 2). It is clear that the P.A. of $45^{\circ}$ lies closest to the actual P.A. of the LS-RCrA 1 outflow as offsets are maximized at this slit position. The change in displacement with P.A. allows the P.A. of the outflow to be constrained. As the slit is moved from $45^{\circ}$ to $82^{\circ}$, displacement decreases from $\sim 145$ mas to 45 mas in the FELs and $\sim 50$ mas to 10 mas in $\mathrm{H} \alpha$. From this, the offset one would expect to measure at P.A.s of $90^{\circ}$ and $0^{\circ}$ is calculated. These two values place the outflow P.A. at $\sim 15^{\circ}$.

Note that while only the blueshifted outflow is traced by the FELs both the blueshifted and redshifted lobes are traced by $\mathrm{H} \alpha$. A similar trend has been seen for some CTTSs and it is hypothesized that while the circumstellar disk obscures the redshifted forbidden emission, the permitted emission (which originates much closer to the star due to its significantly higher critical density) is visible through a hole or gap in the disk (Takami et al. 2001; Whelan et al. 2004). We estimate that a minimum (projected) disk radius of 0 '. 15 ( $\leqslant 22.5 \mathrm{AU}$ at the distance of the Coronae Australis star-forming region) is needed in order to hide the redshifted component to the LS-RCrA 1 forbidden emission. The formation of disk gaps in CTTSs is believed to result from grain growth in the inner disk or clearing due to an orbiting planetesimal (Whelan et al. 2004). For CTTSs and young BDs grain growth is probably the most likely explanation and signals the onset of planet formation (Apai et al. 2005). Previous studies have argued for the occurrence of planet-forming processes in BD disks (Muzerolle et al. 2006; Scholz et al. 2007). The discovery of a dust gap in the disk of LS-RCrA 1, large enough for the redshifted outflow to be detected, supports the hypothesis that BD disks could at some stage harbor planets.

Spectro-astrometric studies of CTTSs and Herbig $\mathrm{Ae} / \mathrm{Be}$ stars with disk dust holes have allowed the radii of the holes to be estimated (Takami et al. 2001; Whelan et al. 2004). The measured redshifted offset is taken as an estimate of the radius of the dust hole. In all cases, estimates of hole radius from spectro-astrometry were compared with estimates made from the spectral energy distributions (SEDs) of the stars. The SEDs of stars with circumstellar disks show an excess of emission above the stellar emission (originating from the disk). Stars with inner disk holes have dips in this excess emission, corresponding to the distance from the star that the region free from dust extends to. Both methods of measuring the radii of the dust holes have yielded similar results (Takami et al. 2003). 
For LS-RCrA 1, a redshifted offset of $45 \pm 14$ mas at $45^{\circ}$ indicates a gap of $\sim 6.75 \pm 2 \mathrm{AU}$ in radius. This is somewhat larger than the gap observed by Muzerolle et al. (2006) in the BD L316 (this estimate was made from the SED of the BD). Analysis of the SED of LS-RCrA 1 and of spectra taken at the outflow P.A. is needed in order to constrain the size of its inner dust hole.

Barrado y Navascués et al. (2004) argue that LS-RCrA 1 has an edge-on disk, explaining its faintness compared to other objects of the same spectral type. For an outflow source with an edge-on disk and thus an outflow in the plane of the sky, one would expect to see both lobes of the outflow and to measure low radial velocities for any lines tracing the outflow. The highand low-velocity components (HVC, LVC) to FELs, commonly detected in the spectra of CTTSs (Hirth et al. 1997), appear blended for high jet inclination angles. A good example is the outflow driven by the BD 2MASS1207-3932. This BD is known to have a near-edge-on disk and the analysis of its outflow supported this. Whelan et al. (2007) detected blueshifted and redshifted [O I] $\lambda 6300$ emission at velocities of $+8 \mathrm{~km} \mathrm{~s}^{-1}$ and $-4 \mathrm{~km} \mathrm{~s}^{-1}$. The lack of redshifted forbidden emission means that LS-RCrA 1 does not have an edge-on disk but that the disk is sufficiently inclined to obscure the red lobe.

There is also some evidence of separation between the lowand high-velocity forbidden emission. The differing origins of the LVC and HVC are well documented for CTTSs and results in $\left[\mathrm{N}_{\mathrm{II}}\right] \lambda 6583$ only tracing the HVC (well-collimated high-velocity jet) and both $[\mathrm{S}$ II] 66731 and [O I] 66300 tracing both the LVC (less-collimated low-velocity wind) and HVC (Hartigan et al. 1995). Although [OI] 26300 due to its much higher critical density is the better tracer of high-density regions close to the star and hence the LVC. The average radial velocities listed above for the [O I] $\lambda 6300,[\mathrm{~N}$ II] 26583 , and [S II] 6731 lines demonstrate that the $[\mathrm{O} \mathrm{I}] \lambda 6300$ line primarily traces the $\operatorname{LVC}\left(-5 \mathrm{~km} \mathrm{~s}^{-1}\right)$, the [N II] $\lambda 6583$ line the HVC only $(-22 \mathrm{~km}$ $\mathrm{s}^{-1}$ and wing extended to $-75 \mathrm{~km} \mathrm{~s}^{-1}$ ), and the [S II] 26731 line the LVC and HVC (peak at $-13 \mathrm{~km} \mathrm{~s}^{-1}$ and wing extended to $-40 \mathrm{~km} \mathrm{~s}^{-1}$ ). This is supported by the spectro-astrometric analysis of the $[\mathrm{S}$ II] $] \lambda 6731$ and $\left[\mathrm{N}_{\text {II }}\right] \lambda 6583$ lines at $\sim 45^{\circ}$. The maximum offset in the $\left[\mathrm{N}_{\mathrm{II}}\right] \lambda 6583$ line occurs at the peak velocity of $\sim-22 \mathrm{~km} \mathrm{~s}^{-1}$; however, for the [S II] $\lambda 6731$ line the maximum offset is in the wing. This points to the fact that the [S II] emission making up the wing originates in the HVC of the outflow, while the bulk of the emission traces a slower, less-extended component.

The LS-RCrA 1 FELs are reminiscent of the FELs found in the spectrum of the CTTS DG Tau. The DG Tau [OI] 6300 and $\left[\mathrm{S}_{\mathrm{II}}\right] \lambda 6731$ lines have a low-velocity primary peak and a high-velocity secondary peak with maximum offsets occurring in the high-velocity peaks. The [O I] $\lambda 6300$ line peaks occur at $\sim-47 \mathrm{~km} \mathrm{~s}^{-1}$ and $-266 \mathrm{~km} \mathrm{~s}^{-1}$ and the [S II] 26731 line peaks at $\sim-47 \mathrm{~km} \mathrm{~s}^{-1}$ and $-240 \mathrm{~km} \mathrm{~s}^{-1}$. The [N II] 6583 line is single peaked and maximum offsets occur at the peak velocity of $-240 \mathrm{~km} \mathrm{~s}^{-1}$. The spectro-astrometric analysis of the DG Tau [O I $] \lambda 6300$ and $\left[\mathrm{S} \mathrm{II}^{\mathrm{I}}\right] \lambda 6731$ lines is published in Whelan et al. (2004). The analysis of the [N II] $] 6583$ was not published but values quoted here come from the same data. Overall the kinematical and spectro-astrometric results do not support the argument that LS-RCrA 1 has an edge-on disk. As a result the questions related to the luminosity of this source persist. The fact that it is a strong accretor is now the most likely explanation of the lack of agreement between its spectral type and luminosity.
Finally, the spectro-astrometric analysis of the $\mathrm{H} \alpha$ line shall be discussed in the context of the use of $\mathrm{H} \alpha$ line widths as a measure of mass accretion in BDs. In addition to being used as a diagnostic of ongoing accretion, model fitting of the $\mathrm{H} \alpha$ line is used to estimate the rate of mass accretion (Muzerolle et al. 2003). The detection of a significant outflow component to the LS-RCrA $1 \mathrm{H} \alpha$ line cautions against the use of $\mathrm{H} \alpha$ alone as a measure of mass accretion rates. Other methods of estimating the mass accretion rate are based on measurements of veiling and of the flux of the Ca II $\lambda 8662$ line (Mohanty et al. 2005). It is clearly important to rule out any significant contribution to the $\mathrm{H} \alpha$ line from a jet/outflow before using it as a reliable probe of magnetospheric accretion.

\section{SUMMARY}

LS-RCrA 1 was the first young BD found to exhibit signatures of outflow activity in the form of FELs, similar to those known to trace CTT jets. Although the [O I] $\lambda \lambda 26300,6363$, $[\mathrm{S}$ II] $] \lambda 6716,6731$, and [N $\mathrm{NI}] \lambda 6583$ lines are strong, spectroscopic and imaging studies failed to find any extension in these line regions in the form of an outflow (Fernández \& Comerón 2005). Furthermore, LS-RCrA 1 which is noted to be faint in comparison to other sources of the same spectral type, was shown to be a remarkably strong accretor. Several papers have attempted to reconcile the strong outflow and infall signatures with the subluminous nature of this object and have offered an edge-on disk scenario as the most likely explanation.

The spectro-astrometric analysis presented here greatly adds to our understanding of this exemplar object as follows. First, a spatial offset in the $\left[\mathrm{S}_{\mathrm{II}}\right] \lambda 6731$ and $[\mathrm{N}$ II] $] \lambda 6583$ FEL regions, from the continuum, is seen, verifying their excitation in a blueshifted outflow and constraining the P.A. of the outflow at $\sim 15^{\circ}$. The velocities and offsets measured in the lines at a P.A. of $45^{\circ}$ (closest to the outflow P.A.) show the lines to be tracing different components outflow. The $[\mathrm{N}$ II] $\lambda 6583$ is clearly tracing the higher-velocity component (associated with a jet in CTTSs), while the $\left[\mathrm{S} \mathrm{II}_{\mathrm{II}}\right] \lambda 6731$ line is a mixture of high- and low-velocity emission and the maximum offsets occur in the blueshifted line wing. The complete lack of redshifted forbidden emission along with some evidence of separation between lowand high-velocity emission means that LS-RCrA 1 cannot have an edge-on disk.

Second, it is demonstrated for the first time that $\mathrm{H} \alpha$ can trace outflows driven by BDs and that the contribution to the line can be significant. This discovery is of consequence as $\mathrm{H} \alpha$ line widths are frequently used as an indicator of accretion activity (10\% width) and a measure of mass accretion rate in BDs. Any study using $\mathrm{H} \alpha$ to probe mass accretion must consider the possibility of a significant outflow component to the line. Spectro-astrometry is the best tool to use to disentangle the outflow component to any permitted emission line. Finally, evidence of a sizeable dust hole in the disk of LS-RCrA 1 exists supporting the hypothesis that BD disks could possibly harbor planets.

Overall, results support the continuation of key processes in the formation and evolution of low-mass stars into the substellar regime. Again it is clear that outflows accompany the formation of BDs and that they are analogous but scaled down from $\mathrm{T}$ Tauri outflows. The established tracers of CTT jets, the optical FELs, probe BD outflows in an equivalent way and can, as demonstrated here, have LVCs and HVCs. In addition, the 
processes leading to the formation of debris disks also appear to occur in the disks of BDs.

The present work was supported in part by the European Community's Marie Curie Actions-Human Resource and Mobility within the JETSET (Jet Simulations, Experiments, and Theory) network under contract MRTN-CT-2004 005592 and by Science Foundation Ireland (contract 04/BRG/P02741). This work is based on observations made with the European Southern Observatory telescopes obtained from the ESO/STECF Science Archive Facility.

\section{REFERENCES}

Apai, D., Pascucci, I., Bouwman, J., Natta, A., Henning, T., \& Dullemond, C. P. 2005, Science, 310,834

Bailey, J. A. 1998, Proc. SPIE, 3355, 932

Barrado y Navascués, D., Mohanty, S., \& Jayawardhana, R. 2004, ApJ, 604 284

Bourke, T. L., Crapsi, A., Myers, P. C., Evans, N. J. II, Wilner, D. J., Huard, T. L., Jørgensen, J. K., \& Young, C. H. 2005, ApJ, 633, L129

Brannigan, E., Takami, M., Chrysostomou, A., \& Bailey, J. 2006, MNRAS, 367, 315

Dekker, H., D’Odorico, S., Kaufer, A., Delabre, B., \& Kotzlowski, H. 2000, Proc. SPIE, 4008, 534

Edwards, S. 2008, arXiv:0809.3603

Fernández, M., \& Comerón, F. 2001, A\&A, 380, 264

Fernández, M., \& Comerón, F. 2005, A\&A, 440, 1119

Hartigan, P., Edwards, S., \& Ghandour, L. 1995, ApJ, 452, 736
Hirth, G. A., Mundt, R., \& Solf, J. 1997, A\&AS, 126, 437

Jayawardhana, R., Mohanty, S., \& Basri, G. 2003, ApJ, 592, 282

Mohanty, S., Jayawardhana, R., \& Basri, G. 2005, ApJ, 626, 498

Muzerolle, J., Hillenbrand, L., Calvet, N., Briceño, C., \& Hartmann, L. 2003, ApJ, 592, 266

Muzerolle, J., et al. 2006, ApJ, 643, 1003

Natta, A., \& Testi, L. 2001, A\&A, 376, L22

Natta, A., Testi, L., Comerón, F., Oliva, E., D’Antona, F., Baffa, C., Comoretto, G., \& Gennari, S. 2002, A\&A, 393, 597

Natta, A., Testi, L., Muzerolle, J., Randich, S., Comerón, F., \& Persi, P. 2004, A\&A, 424, 603

Phan-Bao, N., et al. 2008, arXiv:0810.2588

Ray, T., Dougados, C., Bacciotti, F., Eislöffel, J., \& Chrysostomou, A. 2007, in Protostars and Planets V, ed. B. Reipurth, D. Jewitt, \& K. Keil (Tucson, AZ: Univ. Arizona Press), 231

Scholz, A., \& Jayawardhana, R. 2006, ApJ, 638, 1056

Scholz, A., Jayawardhana, R., Wood, K., Meeus, G., Stelzer, B., Walker, C., \& O’Sullivan, M. 2007, ApJ, 660, 1517

Takami, M., Bailey, J., \& Chrysostomou, A. 2003, A\&A, 397, 675

Takami, M., Bailey, J., Gledhill, T. M., Chrysostomou, A., \& Hough, J. H. 2001, MNRAS, 323, 177

Tout, C. A., Livio, M., \& Bonnell, I. A. 1999, MNRAS, 310, 360

Whelan, E., \& Garcia, P. 2008, Lecture Notes in Physics 742 (Berlin: Springer), 123

Whelan, E. T., Ray, T. P., Bacciotti, F., \& Jayawardhana, R. 2006, New Astron. Rev., 49, 582

Whelan, E. T., Ray, T. P., Bacciotti, F., Natta, A., Testi, L., \& Randich, S. 2005, Nature, 435, 652

Whelan, E. T., Ray, T. P., \& Davis, C. J. 2004, A\&A, 417, 247

Whelan, E. T., Ray, T. P., Randich, S., Bacciotti, F., Jayawardhana, R., Testi, L., Natta, A., \& Mohanty, S. 2007, ApJ, 659, L45

Wilking, B. A., Greene, T. P., \& Meyer, M. R. 1999, AJ, 117, 469 